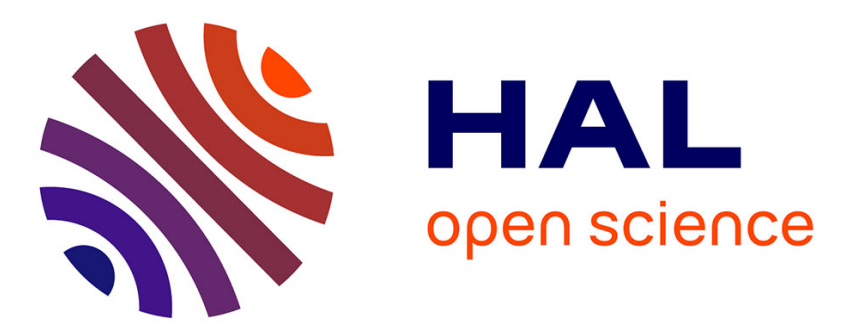

\title{
Prise en compte du rôle des inclusions non métalliques dans le comportement anisotrope en fatigue d'aciers forgés
}

\author{
Etienne Pessard, Franck Morel, Anne Morel
}

\section{To cite this version:}

Etienne Pessard, Franck Morel, Anne Morel. Prise en compte du rôle des inclusions non métalliques dans le comportement anisotrope en fatigue d'aciers forgés. Revue de Métallurgie, 2010, 107 (10-11), pp.427-432. 10.1051/metal/2011006 . hal-00805326

\section{HAL Id: hal-00805326 \\ https://hal.science/hal-00805326}

Submitted on 27 Mar 2013

HAL is a multi-disciplinary open access archive for the deposit and dissemination of scientific research documents, whether they are published or not. The documents may come from teaching and research institutions in France or abroad, or from public or private research centers.
L'archive ouverte pluridisciplinaire HAL, est destinée au dépôt et à la diffusion de documents scientifiques de niveau recherche, publiés ou non, émanant des établissements d'enseignement et de recherche français ou étrangers, des laboratoires publics ou privés. 


\title{
Prise en compte du rôle des inclusions non métalliques dans le comportement anisotrope en fatigue d'aciers forgés
}

\author{
ETIENNE PESSARD ${ }^{\mathrm{a}}$, FRANCK MOREL ${ }^{\mathrm{a}}$, ANNE MOREL $^{\mathrm{a}}$ \\ ${ }^{a}$ Arts et Métiers ParisTech, Angers, France
}

\begin{abstract}
Résumé: L'objectif de cette étude est de proposer un critère de fatigue anisotrope pour le dimensionnement en fatigue de pièces industrielles forgées. Des résultats de différentes campagnes d'essais obtenus sur 3 nuances d'acier laminés sont tout d'abord présentés. Une attention particulière est portée à l'observation et à l'analyse des mécanismes d'amorçage à l'origine du comportement anisotrope en fatigue. Un critère de fatigue probabiliste permettant de tenir compte de la compétition possible entre différents types de mécanismes d'amorçage est ensuite développé. Ce critère permet notamment de tracer un diagramme de type Kitagawa probabiliste.
\end{abstract}

\section{INTRODUCTION}

Les matériaux laminés ou forgés sont reconnus pour leur bonne tenue mécanique en particulier en fatigue. Cette caractéristique est liée à l'amélioration de la compacité et à l'obtention d'une microstructure plus fine par l'opération de mise en forme. Le fibrage est le terme communément employé pour décrire l'anisotropie d'un matériau forgé, il définit une orientation préférentielle selon laquelle la microstructure ou les défauts présents dans le matériau ont été déformés par l'opération de mise en forme.

Ce fibrage est responsable du caractère anisotrope de la tenue en fatigue, cette variation peut être de l'ordre de $35 \%$ pour un acier $42 \mathrm{CrMo} 4$ [1] ou de $15 \%$ pour un acier inoxydable [2]. Certains logiciels de mise en forme permettent actuellement de prédire l'orientation de ce fibrage. L'objectif de cet article est de proposer un critère de fatigue permettant de tenir compte simplement de cette anisotropie via la prise en compte du fibrage induit par l'opération de mise en forme.

\section{MISE EN EVIDENCE DU COMPORTEMENT ANISOTROPE DES ACIERS LAMINES}

Afin de proposer un critère de fatigue adapté à une large gamme d'acier, 3 nuances différentes ont été sélectionnées.

La première nuance étudiée est un acier bainitique : le Metasco MC (Tableau I). Le comportement anisotrope en fatigue de ce premier matériau sera étudié en détail. Cette première partie expérimentale permettra notamment de mettre en évidence le rôle des inclusions allongées sur le comportement anisotrope en 
fatigue d'un acier laminé dont la microstructure est composée de grains équiaxes.

L'objectif de la seconde partie expérimentale sera de définir si la présence d'une microstructure présentant une morphologie de grains allongés peut être à l'origine d'un comportement anisotrope en fatigue. Pour répondre à cette question, deux nuances d'aciers laminés, le C35 et le Splitasco ayant la particularité de présenter le même type de microstructure biphasée (ferritoperlitique) mais des niveaux de résistance mécanique différents seront également testés (Tableau I).

Tableau I. Caractéristiques mécaniques des aciers étudiés

\begin{tabular}{ccccc}
\hline Matériau & Microstructure & $\mathrm{Rm}(\mathrm{MPa})$ & $\mathrm{Re}_{0,2 \%}(\mathrm{MPa})$ & $\sigma_{\mathrm{D}(\mathrm{MPa})}$ \\
\hline Metasco MC & $\begin{array}{c}\text { Bainitique } \\
\text { Grains équiaxes }\end{array}$ & 1150 & 750 & 476 \\
\hline Splitasco & $\begin{array}{c}\text { Ferrito-perlitique } \\
\text { Grains allongés }\end{array}$ & 1050 & 750 & 420 \\
\hline $\mathrm{C} 35$ & $\begin{array}{c}\text { Ferrito-perlitique } \\
\text { Grains allongés }\end{array}$ & 580 & 350 & 230 \\
\hline
\end{tabular}

\section{Comportement anisotrope en fatigue de l'acier Metasco MC.}

Le premier matériau étudié est un acier bainitique : le Metasco MC. Ce matériau laminé avec un rapport de corroyage de l'ordre de 15 possède des inclusions de sulfure de manganèse allongées dans le sens de laminage. Des observations optiques ainsi que la réalisation de figures de pôles ont permis de montrer que sa microstructure est isotrope. Si le Metasco MC possède un comportement en fatigue anisotrope cela ne peut être dû qu'à la présence de ses inclusions allongées.

Des éprouvettes ont été prélevées dans trois directions différentes à $0^{\circ}, 45^{\circ}$ et $90^{\circ}$ (Figure 1). 15 éprouvettes par sens de prélèvement ont été testées en tractioncompression $\mathrm{R}=-1$ en suivant la méthode staircase avec un seuil fixé à $\mathrm{N}=2.10^{6}$ cycles.

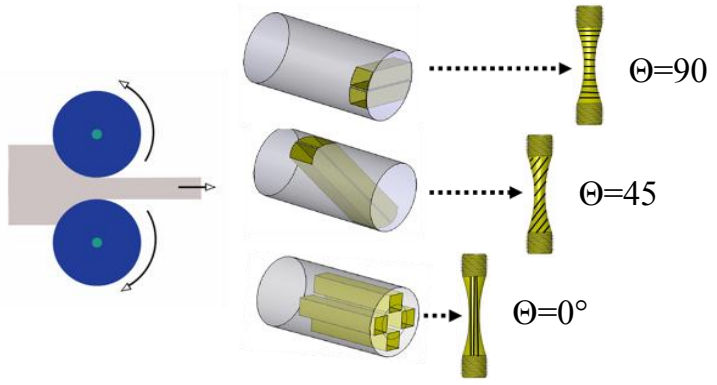

Figure 1 Orientation des différents prélèvements

L'évolution de la limite d'endurance en fonction de l'orientation du fibrage représentée sur la Figure 4 est décroissante entre $0^{\circ}$ et $90^{\circ}$. Les observations de 
propagation de fissure en surface montrent que pour un fibrage orienté à $0^{\circ}$ toutes les fissures s'amorcent au sein de la matrice et sont orientées avec un angle de $45^{\circ}$ par rapport à la contrainte appliquée.

Pour un fibrage orienté à $90^{\circ}, 2$ types d'amorçage sont observés ; certaines fissures s'amorcent à partir de la matrice et sont orientées à $45^{\circ}$, d'autres s'amorcent à partir d'amas d'inclusions et sont orientées à $90^{\circ}$ par rapport à la contrainte appliquée (Figure 2 a),b)).

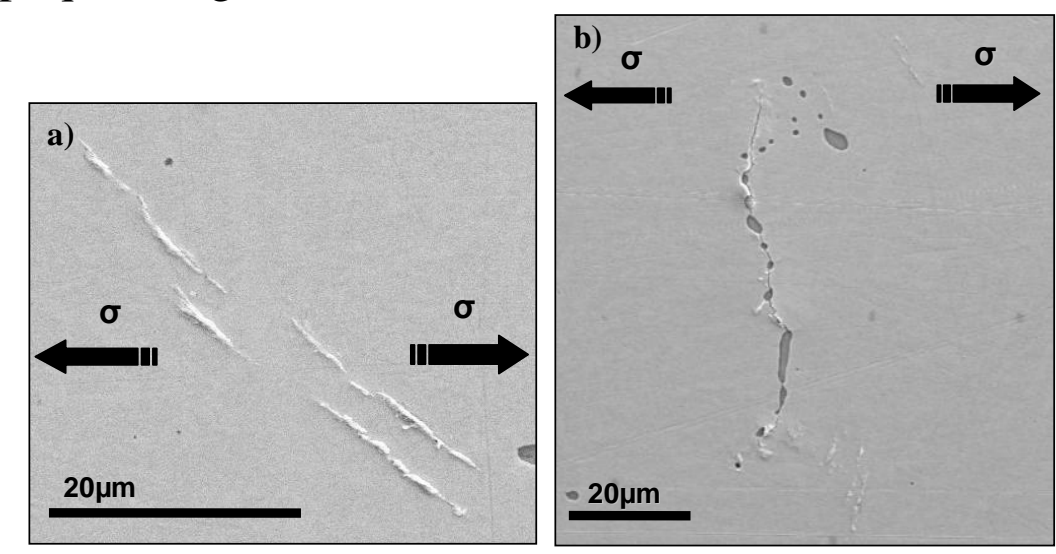

Figure 2 Suivi du trajet de fissure en surface, fibrage orienté à $90^{\circ}$, fissure amorcée à partir a) de la matrice, b) d'une bande d'inclusions

\section{Comportement anisotrope en fatigue de l'acier C35.}

Le second matériau étudié est un acier ferrito-perlitique mi-dur : le C35. Ce matériau laminé possède une microstructure en bandes et des inclusions de sulfure de manganèse allongées dans le sens de laminage (Figure 3). Flacelière [3] a montré que la tenue en fatigue de l'acier C35 en traction-compression est la même pour un prélèvement orienté dans le sens longitudinal (fibrage orienté à $0^{\circ}$ ) et pour un prélèvement orienté dans le sens transversal (fibrage orienté à $90^{\circ}$ ). Les inclusions ne semblent donc pas à l'origine d'un comportement anisotrope en fatigue. Les observations de mécanismes réalisées n'ont pas non plus pas révélé de différences notables de conditions d'amorçage ou de propagation pour ces deux orientations de fibrage.

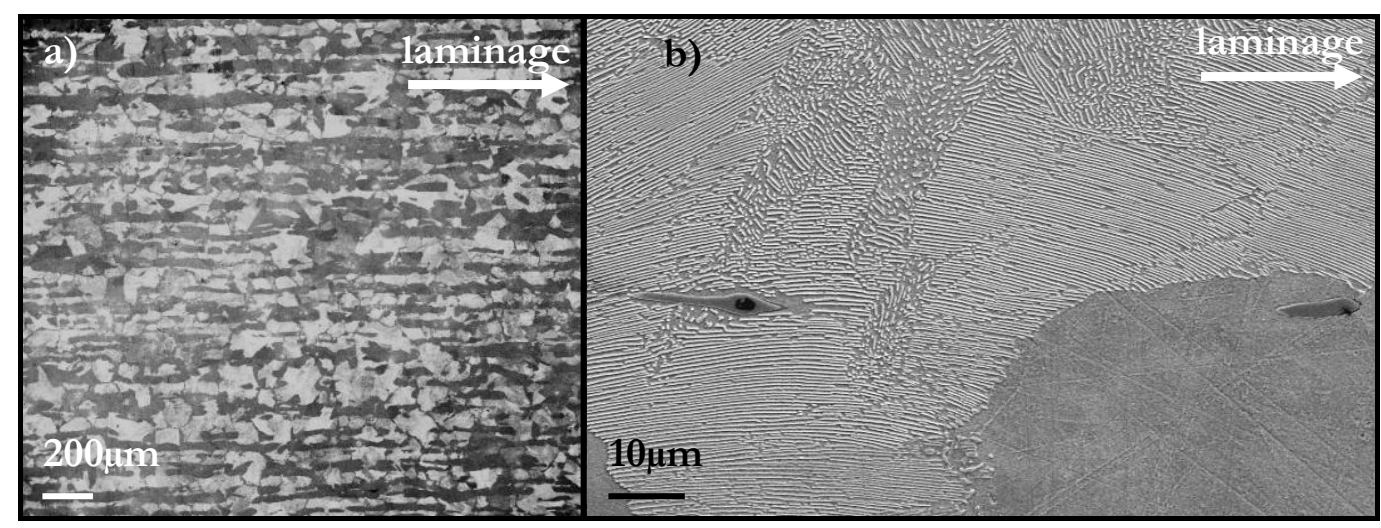

Figure 3 Microstructure ferrito-perlitique en bandes de l'acier C35

Afin de déterminer rapidement la limite d'endurance en traction-compression, la méthode des paliers successifs (Locati) a été appliquée sur deux éprouvettes prélevées à 45 et $90^{\circ}$ par rapport à l'axe de la barre $(\varnothing 70 \mathrm{~mm})$. Les résultats 
obtenus prédisent des limites d'endurance très proches pour des orientations de fibrage à 45 et $90^{\circ}$. A partir des résultats de Flacelière et de ces nouvelles informations, nous pouvons conclure que l'acier C35 possède un comportement en fatigue isotrope malgré la présence d'inclusions allongées et d'une microstructure constituée de grains allongés (Figure 4).

\section{Comportement anisotrope en fatigue de l'acier Splitasco.}

Le troisième matériau étudié est le Splitasco. Cette nuance, développée pour les bielles sécables, possède comme l'acier C35 une microstructure ferritoperlitique en bandes et des caractéristiques mécaniques légèrement inférieures au Metasco MC (Tableau I).Tout comme le Metasco MC, le Splitasco possède des inclusions de $\mathrm{MnS}$ allongées dans le sens de laminage. Celles-ci sont plus massives que dans le Metasco MC car elles possèdent un diamètre moyen d'environ $4 \mu \mathrm{m}$ contre $2 \mu \mathrm{m}$ pour le Metasco MC. L'objectif étant toujours de caractériser le comportement anisotrope du Splitasco, des éprouvettes ont été prélevées à 0,45 et $90^{\circ}$ par rapport à l'axe d'une barre laminée $(\varnothing 70 \mathrm{~mm})$. La méthode staircase a été appliquée afin de déterminer la limite d'endurance pour chacune des directions de prélèvement.

La limite d'endurance en traction-compression de l'acier Splitasco est anisotrope et chute continûment avec l'augmentation de l'angle de fibrage (Figure 4).

\section{Bilan des essais de fatigue}

Les essais de fatigue réalisés sur l'acier C35 ferrito-perlitique permettent de conclure que, pour ce matériau, la limite d'endurance en traction alternée est isotrope. Malgré la présence d'inclusions de sulfure de manganèse allongées selon l'axe de laminage, l'amorçage est toujours localisé au niveau de la matrice et ce quelle que soit l'orientation du fibrage par rapport à l'axe de sollicitation. Le fait que l'amorçage n'ait pas lieu au niveau des inclusions n'est pas surprenant et est en accord avec le diagramme de Kitagawa qui, pour un matériau présentant des caractéristiques de résistance peu élevées, associe une taille de défaut critique élevée. En revanche, il est étonnant que l'orientation privilégiée des barrières microstructurales ne génère pas une tenue en fatigue anisotrope. On peut imaginer que l'orientation des barrières microstructurales présentes dans cette microstructure ferrito-perlitique a un effet limité sur la phase d'amorçage. Les barrières ferrito-perlitiques apparaissent peut-être également à une échelle trop grande par rapport à une taille de fissure amorcée dans un grain ferritique.

Le comportement en fatigue des aciers Metasco et Splitasco en traction alternée est anisotrope et très proche de celui observé pour le Metasco MC. Pour ces matériaux possédant une limite d'endurance plus élevée que le C35, il est cohérent d'observer, pour un fibrage orienté à $90^{\circ}$, un amorçage localisé au niveau des inclusions, la taille de défaut critique étant plus faible (diagramme de Kitagawa). Tout comme pour le C35, l'orientation privilégiée des barrières microstructurales ne semble pas avoir d'effet sur le comportement en fatigue. 
Pour les trois nuances d'acier étudiées dans le domaine de l'endurance illimitée (Metasco MC, Splitasco et C35) les comportements observés en fatigue sont les mêmes, la matrice est isotrope et seule la présence d'inclusions peut permettre de justifier le caractère anisotrope de la tenue en fatigue. Cette dernière conclusion est primordiale et constituera l'une des hypothèses majeures de la modélisation proposée dans la prochaine partie.

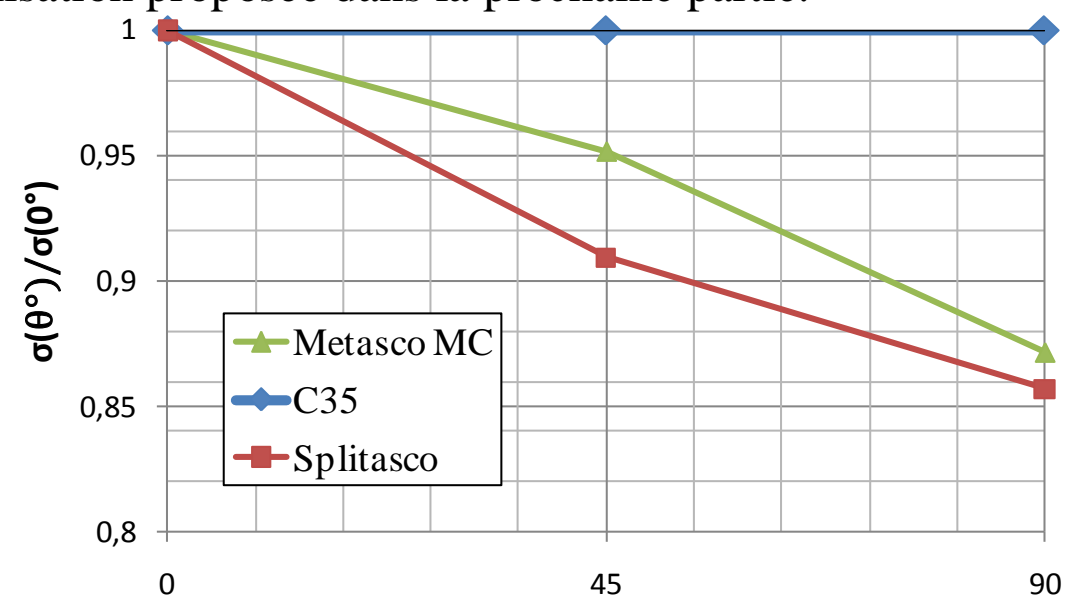

Angle du fibrage en degrés

Figure 4 Évolution de la limite d'endurance en traction/compression $\mathrm{R}=-1$ en fonction de l'orientation du fibrage pour les matériaux étudiés

\section{MODELISATION PROPOSEE}

Les observations de mécanisme en surface ont montré que l'anisotropie de la tenue en fatigue des aciers étudiés est liée à une compétition entre deux types de mécanismes. Lorsque le fibrage est orienté à $0^{\circ}$ les défauts (i.e. les amas d'inclusion) sont peu nocifs et l'amorçage apparaît au sein de la matrice. Pour un fibrage orienté à $90^{\circ}$ deux types d'amorçage sont en compétition, l'amorçage peut naitre au sein de la matrice ou à partir d'amas d'inclusion.

Dans le domaine de la fatigue des matériaux métalliques, deux grandes catégories de modélisation coexistent. La première considère que l'amorçage d'une fissure est lié à la plasticité locale d'un grain isolé ou mal orienté. La seconde se base sur la mécanique linéaire de la rupture pour décrire la propagation d'une fissure préexistante. Le choix qui est fait ici est de combiner ces deux approches par une approche probabiliste.

La probabilité de rupture $\mathrm{P}_{\mathrm{F} 1}$ associée à un amorçage possible au sein de la matrice est prise en compte via l'approche de Morel [4]. Cette approche se base sur les hypothèses d'adaptation élastique à l'échelle mésoscopique et du maillon le plus faible appliqué sur l'ensemble des plans d'un volume élémentaire représentatif.

Cette probabilité peut s'exprimer selon l'équation (1). 


$$
P_{F 1}=1-\exp \left[-\frac{1}{V_{01}} \int_{V}\left\{\int_{\varphi=0}^{2 \pi} \int_{\theta=0}^{\pi} \int_{\psi=0}^{2 \pi}\left(\frac{T_{a}(\varphi, \theta, \psi)}{T_{01}}\right)^{m_{1}} \sin \theta d \psi d \theta d \varphi\right\} d V\right]
$$

avec $T_{a}(\varphi, \theta, \psi)$ : amplitude de cission sur un plan défini par $(\varphi, \theta, \psi)$ et $\mathrm{m}_{1}$ exposant lié à la dispersion associée au $1^{\mathrm{er}}$ mécanisme.

L'intégration dans l'équation (1) permet de prendre en compte le potentiel d'amorçage sur l'ensemble des plans d'un élément de volume.

La probabilité de propagation d'une fissure à partir d'un amas d'inclusion $\mathrm{P}_{\mathrm{F} 2}$ est prise en compte en se basant sur la Mécanique Linéaire de la Rupture. Les amas d'inclusions sont alors assimilés à des fissures préexistantes.

Nous supposons ici que pour des fissures longues, le mode I de propagation est dominant. En considérant les défauts comme des fissures elliptiques, la théorie de la mécanique de la rupture permet d'estimer le facteur d'intensité de contrainte $\Delta K$ en fonction de l'amplitude des contraintes appliquées, du facteur de forme et de la taille de la fissure. La condition de non rupture est définie en comparant le facteur d'intensité de contrainte calculé sur la structure $\Delta K$ au seuil de propagation de fissure du matériau $\Delta K_{t h}$.

Il y a non rupture si :

$$
\Delta K<\Delta K_{t h}
$$

Le caractère aléatoire du seuil de propagation est introduit par une distribution de Weibull. L'originalité de cette approche réside dans le fait que nous proposons de considérer un facteur d'intensité de contrainte locale $\Delta K(\theta, \varphi)$. Sur un plan défini par les angles $(\theta, \varphi)$, la probabilité de dépasser le seuil de propagation de fissure s'écrit comme la probabilité de trouver une valeur critique $\Delta K_{t h}$ inférieure à la valeur appliquée $\Delta K(\theta, \varphi)$ :

$$
P_{02}\left(\Delta K_{t h}<\Delta K(\theta, \varphi)\right)=\int_{\Delta K(\theta, \varphi)}^{+\infty} f_{02}\left(\Delta K_{t h}\right) d \Gamma_{C}
$$

$\mathrm{Si} \mathrm{m}_{2}$ gouverne la dispersion et le facteur $\Delta K_{t h 02}$ la valeur moyenne de la distribution du seuil de propagation de fissure, la probabilité de rupture sur un plan devient :

$$
P_{02}(\theta, \varphi)=1-\exp \left[-\left(\frac{\Delta K(\theta, \varphi)}{\Delta K_{t h 02}}\right)^{m_{2}}\right]
$$

Considérer la propagation d'une fissure à partir d'un défaut uniquement dans le plan où la contrainte normale est maximale est assez restrictif. Si cette modélisation donne a priori de bons résultats pour un chargement simple de traction alternée, il nous semble que dans le cas de chargement multiaxiaux cette approche est rapidement limitée. Afin de mieux prendre en compte la spécificité $\mathrm{du}$ chargement, nous proposons d'estimer la possibilité d'amorçage sur l'ensemble des plans de fissuration potentiels en faisant la somme des 
probabilités de propagation sur l'ensemble des plans du Volume Élémentaire Représentatif. En appliquant l'hypothèse du maillon le plus faible sur tous les plans d'un volume de référence $\mathrm{V}_{0}$, la probabilité de propagation de la fissure au sein de ce volume peut s'écrire :

$$
P_{F 02}=P\left(\Delta K_{t h}<\Delta K(\theta, \varphi)\right)=1-\exp \left[-\frac{1}{D_{02}} \int_{\varphi=0}^{2 \pi} \int_{\theta=0}^{\pi}\left(\frac{\Delta K(\theta, \varphi)}{\Delta K_{t h 02}}\right)^{m_{2}} \sin \theta d \theta d \varphi\right]
$$

La probabilité de rupture totale de la structure peut être obtenue en appliquant une seconde fois l'hypothèse du maillon le plus faible. La somme des probabilités de rupture de chacun des volumes élémentaires peut s'écrire :

$$
P_{F 2}=1-\exp \left[-\frac{1}{V_{02}} \int_{V}\left\{\int_{\varphi=0}^{2 \pi} \int_{\theta=0}^{\pi}\left(\frac{\Delta K(\theta, \varphi)}{\Delta K_{t h 02}}\right)^{m_{2}} \sin \theta d \theta d \varphi\right\} d V\right]
$$

Le coefficient de concentration de contrainte locale $\Delta K(\theta, \varphi)$ peut être déterminé pour chacun des plans par l'expression :

$$
\Delta K(\theta, \varphi)=\frac{\sigma_{a}(\theta, \varphi) \sqrt{\pi a}}{\Phi(\theta)}
$$

avec $\sigma_{a}(\varphi, \theta)$ amplitude de la contrainte normale appliquée sur un plan défini par $(\varphi, \theta)$.

Notons que la géométrie du défaut sur chacun des plans est introduite via la définition du facteur de forme $\Phi(\theta)$.

En utilisant l'expression (7), la probabilité de rupture de la structure devient :

$$
P_{F 2}=1-\exp \left[-\frac{1}{V_{02}} \int_{V}\left\{\int_{\varphi=0}^{2 \pi} \int_{\theta=0}^{\pi}\left(\frac{\sigma_{a}(\varphi, \theta) \sqrt{\pi a}}{\Phi(\theta) \Delta K_{t h 02}}\right)^{m_{2}} \sin \theta d \theta d \varphi\right\} d V\right]
$$

La probabilité de survie du composant est définie en appliquant l'hypothèse du maillon le plus faible. Elle est obtenue en procédant au produit des deux probabilités de survie relatives aux deux types de mécanismes observés :

$$
1-P_{F}=\left(1-P_{F 1}\right)\left(1-P_{F 2}\right)
$$




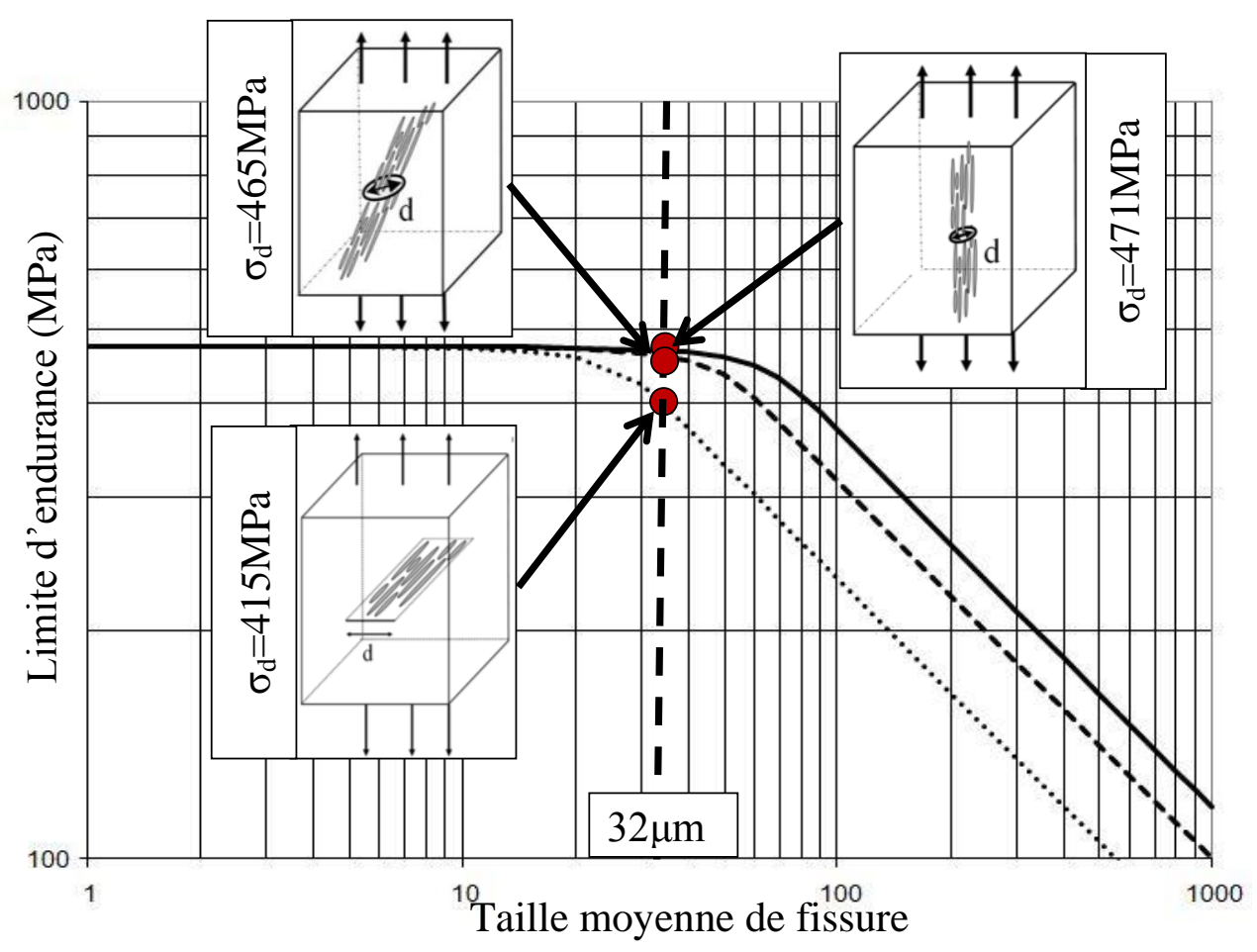

Figure 5 Diagramme de Kitagawa obtenu pour chaque orientation de fibrage

En faisant varier la taille du défaut dans l'équation (9), l'évolution de la limite d'endurance en fonction de la taille du défaut pour différentes orientations du fibrage peut être tracée (Figure 5). La figure obtenue est très proche du critère de Kitagawa et permet de prendre en compte la compétition possible entre deux types de mécanismes.

En connaissant la taille moyenne des défauts considérés présents au sein du matériau, ces diagrammes permettent de prédire la limite d'endurance pour différentes orientations de fibrage par rapport à la contrainte appliquée.

\section{CONCLUSION}

Des essais de fatigue sur différentes nuances d'acier ont permis de mettre en évidence le rôle des inclusions allongées dans le sens de laminage sur l'anisotropie en fatigue.

L'orientation privilégiée des barrières microstructurales présentes au sein de l'acier Splitasco (ferrito-perlitique) ou de l'acier Metasco (bainitique) ne semble pas avoir d'effet sur le comportement en fatigue.

Des essais en traction compression, $\mathrm{R}=-1$ ont montré, pour les 2 nuances d'aciers anisotropes en fatigue étudiées, que la compétition entre deux types d'amorçage est à l'origine de l'anisotropie. Pour ces deux nuances, la limite d'endurance est maximale pour un fibrage orienté à $0^{\circ}$, et minimale lorsqu'il est orienté à $90^{\circ}$.

Une analyse probabiliste combinant deux types de critères décrivant des mécanismes différents permet de prendre en compte cette compétition et de prédire la limite d'endurance en fonction de l'orientation et de la taille des défauts. 


\section{REMERCIEMENTS}

Ce travail a été réalisé dans le cadre du projet Optiforge soutenu par l'Agence National de la Recherche. Ces résultats sont le fruit d'un partenariat entre industriels (Ascoforge, Ascometal, Cetim, PSA, Setforge, Transvalor) et laboratoires universitaires (INSA Lyon-MATEIS, ENSMP-CEMEF, Arts et Métiers PARISTECH Angers-LAMPA).

\section{REFERENCES}

[1] J.Y. Berard, K. Dang Van and G. Baudry, influence de l'anisotropie sur le comportement en fatigue multiaxiale d'un acier de construction mécanique, IRSID, Technical Note No RI 93062 (1993).

[2] A.Mateo, L. Llanes, N. Akdut, J Stolarz and M.Abglada, Anisotropy effects on the fatigue behaviour of rolled duplex stainless steels, Int. J. of Fatigue, 25, (2003), 481-488.

[3] L. Flacelière, Contribution à la modélisation du dommage en fatigue multiaxiale d'un acier C36-confrontation à l'expérience, Thèse de doctorat, Poitiers

[4] F. Morel, N. Huyen, Plasticity and damage heterogeneity in fatigue, Theoretical and Applied Fracture Mechanics 49 (2008), pp. 98-127. 